\title{
List of Participants
}

Akujor C. E. Nuffield Radio Astronomy Observatory GB-Macclesfield, Cheshire SK11 9DL, U.K. Albrecht P. Universitäts-Sternwarte D-37083 Göttingen, Germany

Alexander T. School of Physics and Astronomy Tel-Aviv University Tel-Aviv 69978, Israel

Aller H. D. Astronomy Department University of Michigan Ann Arbor, MI 48109-1090, U.S.A.

Aller M. F. Astronomy Department University of Michigan Ann Arbor, MI 48109-1090, U.S.A.

Alloin D. Observatoire de Paris-Meudon DAEC, 5 Place Jules Janssen, F-92195 Meudon Cedex, France

Almaini O. Dept. of Physics University of Durham GB-Durham, DH1 3LE, U.K.

Altschuler D. R. Arecibo Observatory, P.O. Box 995, Arecibo 00613, Puerto Rico

Antonucci R. Space Telescope Science Institute Baltimore, MD 21218, U.S.A.

Appl S. Landessternwarte Königstuhl D-69117 Heidelberg, Germany

Aretxaga I. Dpto. de Física Teórica, C-XI. Universidad Autónoma de Madrid. E-28049 Madrid, Spain

Arp H. C. Max-Planck-Institut für Astrophysik D-80802 Garching, Germany

Auh B. Yuseonggu Daejeon, 303-348, Korea

Bảāth L. Onsala Space Observatory Göteborg University S-43992 Onsala, Sweden

Babadzhanyants M. Astronomical Institute St. Petersburg University SU-198904 St. Petersburg, Russia

Bade N. Hamburger Sternwarte Universität Hamburg, Gojenbergsweg 112, D-21029 Hamburg, Germany

Baker A. C. Institute of Astronomy, Madingley Road GB-Cambridge, CB3 OHA, U.K.

Baker J. C. Dept. of Astrophysics The University of Sydney Sydney, NSW 2006, Australia

Balbus S. A. University of Virginia Charlotteville, VA 22903-0818, U.S.A.

Balucinska-Church M. School of Physics \& Space Research, Birmingham Univ., Birmingham B15 2TT, U.K.

Band D. L. Center for Astrophysics \& Space Sciences La Jolla, CA 92093-0111, U.S.A.

Baring M. G. NASA-Goddard Space Flight Center, LHEA, Greenbelt, MD 20771, U.S.A.

Barvainis R. Haystack Observatory Westford, MA 01886, U.S.A.

Baryshev Y. Astronomical Institute St. Petersburg University SU-198904 St. Petersburg, Russia

Bednarek W. Max-Planck-Institut für Kernphysik D-6900 Heidelberg, Germany

Bel N. Observatoire de Paris-Meudon F-92195 Meudon Cedex, France

Bergeron J. A. Institut d'Astrophysique F-75014 Paris, France

Bi H. Beijing Astronomical Observatory Chinese Academy of Sciences Beijing, 100080, China

Bjoernsson C. Stockholm Observatory S-13336 Saltsjöbaden, Sweden

Blecha A. Geneva Observatory 1290 Sauverny, Switzerland

Blom J. J. SRON-Leiden 2333 CA Leiden, The Netherlands

Bochkarev N. Sternberg Astronomical Institute 119899 Moscow, Russia

Boksenberg A. Royal Greenwich Observatory GB-Cambridge, CB3 OEZ, U.K.

Bond I. A. Institute of Phys. and Chem. Res. Cosmic Radiation Laboratory Wako, Saitama 351-01, Japan

Bondi M. Nuffield Radio Astronomy Laboratories Jodrell Bank GB-Macclesfield, SK11 9DL, U.K.

Borgeest U. Hamburger Sternwarte Universität Hamburg D-2050 Hamburg 80, Germany

Bower G. A. Space Telescope Science Institute Baltimore, MD 21218, U.S.A.

Bowman M. Univ. of Manchester Nuffield Radio Astron. Lab. GB-Macclesfield, Cheshire SK11 9DL, U.K.

Bregman J. N. Astronomy Department University of Michigan Ann Arbor, MI 48109-1090, U.S.A.

Bremnes T. R. University of Tromso, Astrophysics Group, P.O. Box 953, 9001 Tromso, Norway

Brinkmann W. Max-Planck-Institut für Extraterrestrische Physik D-85740 Garching, Germany

Brinks E. National Radio Astronomy Observatory Socorro, NM 87801, U.S.A.

Britzen S. Max-Planck-Institut für Radioastronomie D-5300 Bonn 1, Germany

Brunner H. Astronomisches Institut Universität Tübingen D-7400 Tübingen, Germany

xxi 
Buehler P. Observatoire de Genève 1290 Sauverny,

Burbidge G. Center for Astrophysics and Space Sciences La Jolla, CA 92093-0111, U.S.A.

Burbidge M. Center for Astrophysics and Space Sciences La Jolla, CA 92093-0111, U.S.A.

Camenzind M. Landessternwarte Königstuhl D-699117 Heidelberg, Germany

Cappi M. Università di Bologna Dipartimento di Astronomia I-40126 Bologna, Italy

Carone T. E. Space Sciences Laboratory University of California Berkeley, CA 94720, U.S.A.

Carvalho J. C. E-08013 Barcelona, Spain

Catch pole R. M. Royal Greenwich Observatory GB-Cambridge, CB3 OEZ, U.K.

Celotti A. Institute of Astronomy, Madingley Road GB-Cambridge, CB3 OHA, U.K.

Chakrabarti S. K. Tata Institute of Fundamental Research Bombay 400005 , India

Chatterjee T. K. Facultad de Ciencas Fisico-Matemáticas Universidad Autónoma de Puebla Puebla, Mexicı

Chu Y. International Center for Theoretical Physics (ICTP) I-34100 Trieste, Italy

Chun M. Dept. of Astronomy Yonsei University Seoul 120-749, Korea

Churazov E. Russian Academy of Sciences Space Research Institute 117810 Moscow, Russia

Church M. J. School of Physics \& Space Research, Univ. of Birmingham GB-Birmingham B15 2TT, U.K.

Chyzy K. T. Astronomical Observatory Jagiellonian University 30-244 Krakow, Poland

Cid Fernandes R. Institute of Astronomy University of Cambridge GB-Cambridge, CB3 OHA, U.K.

Cimatti A. European Southern Observatory D-8046 Garching, Germany

Clavel J. European Space Agency ESTEC Code SAJ NL-2200 AG Noordwijk, The Netherlands

Clements D. Astrophysics Oxford University GB-Oxford, OX1 2RH, U.K.

Collin-Souffrin S. Institut d'Astrophysique de Paris F-75014 Paris, France

Contini M. School of Physics and Astronomy Tel-Aviv University Tel-Aviv 69978, Israel

Courvoisier T. Observatoire de Genève 1290 Sauverny, Suisse

Cruz-Gonzalez I. Instituto de Astronomia, UNAM Ciudad Universitaria Mexico, D.F. 04510, Mexico

Czerny B. Nicolaus Copernicus Astronomical Center PL-00716 Warszawa, Poland

Dallacasa D. Radiosterrenwacht Dwingeloo NL-7990 AA Dwingeloo, The Netherlands

Di Serego Alighieri S. Osservatorio Astrofisico di Arcetri I-50125 Firenze, Italy

Dietrich M. Universitäts-Sternwarte D-3400 Göttingen, Germany

Doerrer T. Astronomisches Institut Universität Tübingen D-7400 Tübingen, Germany

Dubois P. Observatoire de Strasbourg F-67000 Strasbourg, France

Dultzin-Hacyan D. Instituto de Astronomia, Univ. Nac. Auton. de Mexico Mexico, D.F. 04510, Mexico

Edelson R. NASA Goddard Space Flight Center Code 666 Greenbelt, MD 21230 , U.S.A.

Ekejiuba I. E. Dept. of Physics \& Astronomy Georgia State University Atlanta, GA 30303-308, U.S.A.

Elvis M. Harvard-Smithsonian Center for Astrophysics Cambridge, MA 02138, U.S.A.

Engels D. Hamburger Sternwarte Universität Hamburg D-2050 Hamburg 80, Germany

Falomo R. Osservatorio Astronomico di Padova I-35122 Padova, Italy

Fedorenko V. A.F. Ioffe Physical Technical Institute SU-194021 St. Petersburg, Russia

Ferrarese L. Space Telescope Science Institute Baltimore, MD 21218, U.S.A.

Ferreira J. Laboratoire d'Astrophysique Observatoire de Grenoble F-38041 Grenoble Cedex, France

Ferruit P. Observatoire de Lyon F-69561 St Genis Laval Cedex, France

Field G. Osservatorio Astrofisico di Arcetri I-50125 Firenze, Italy

Fraix-Burnet D. Laboratoire d'Astrophysique F-31400 Toulouse, France

Francis P. J. Steward Observatory University of Arizona Tucson, AZ 85721, U.S.A.

Friedli D. Observatoire de Genève 1290 Sauverny,

Friedrich P. Astronomisches Institut Universität Tübingen D-7400 Tübingen, Germany

Friedrich S. Astronomisches Institut Universität Tübingen D-72076 Tübingen, Germany

Fruscione A. Center for EUV Astrophysics University of California Berkeley, CA 94720, U.S.A. 
Gabuzda D. Dept. of Physics and Astronomy University of Calgary Calgary, AB T2N 1N4, Canada

Ghisellini G. Observatory of Torino I-10025 Pino Torinese, Italy

Ghosh K. K. Indian Inst. of Astrophysics Vainu Bappu Observatory Alangayam, N.A.A., TN 635701 , India

Ghosh T. Arecibo Observatory Arecibo, 00613, Puerto Rico

Giommi P. ESIS / ESRIN I-00044 Frascati, Italy

Gondhalekar P. Astrophysics Group Rutherford Appleton Laboratory Chilton, OXON, OX11 0QX, U.K.

Green A. Department of Physics The University GB-Southampton, SO9 5NH, U.K.

Greenhouse M. A. Lab. for Astrophys. MRC321 Nation. Air \& Space Museum Washington, DC 20560, U.S.A.

Grupe D. Universitaets-Sternwarte Göttingen D-3400 Göttingen, Germany

Haehnelt M. Ecole d'Eté de Physique Théorique F-74310 Les Houches, France

Hagen-Thorn V. A. Astron. Observatory St. Petersburg State Univ. SU-198904 St. Petersburg, Russia

Harris D. MS-3, Center for Astrophysics Cambridge, MA 02138, U.S.A.

Heidt J. Landessternwarte Heidelberg D-6900 Heidelberg, Germany

Heinrich O. M. Institut für Theoretische Astrophysik D-6900 Heidelberg, Germany

Henri G. Laboratoire d'Astrophysique Observatoire de Grenoble F-38041 Grenoble Cedex, France

Hjelm M. Stockholm Observatory S-13336 Saltsjöbaden, Sweden

Ho L. C. Dept. of Astronomy University of California Berkeley, CA 94720, U.S.A.

Howard S. BDM - 409 Washington, DC 20024, U.S.A.

Huang K. Dept. of Physics Nanjing Normal University Nanjing 210024, China

Hughes D. H. Astrophysics Oxford University GB-Oxford, OX1 3RH, U.K.

Hughes P. A. Astronomy Department University of Michigan Ann Arbor, MI 48109-1090, U.S.A.

Hure J. M. Observatoire de Meudon DAEC F-92195 Meudon Principal Cedex, France

Hutchings J. B. Dominion Astrophysical Observatory Victoria, B.C. V8X 4M6, Canada

Ijjima T. Osservatorio Astrofisico di Asiago I-36012 Asiago (Vicenza), Italy

Inoue $H$. Institute of Space and Astronautical Science Kanagawa 229, Japan

Jackson N. Sterrewacht Leiden 2300 RA Leiden, Netherlands

Jannuzi B. Institute for Advanced Study Princeton, NJ 08540, U.S.A.

Jourdain E. Centre d'Etude Spatiale des Rayonnements F-31089 Toulouse Cedex, France

Köhler T. Hamburger Sternwarte Universität Hamburg D-2050 Hamburg 80, Germany

Kaastra J. S. SRON Leiden 2300 RA Leiden, The Netherlands

Kallman T. NASA-Goddard Space Flight Center, LHEA, Greenbelt, MD 20771, U.S.A.

Kembhavi A. Inter-University Centre for Astronomy and Astrophysics Pune 41107, India

Kikuchi S. National Astronomical Observatory Tokyo, 181, Japan

Ko Y. Laboratory for High Energy Astrophysics Greenbelt, MD 20771, U.S.A.

Kollatschny W. Universitäts-Sternwarte D-3400 Göttingen, Germany

Komberg B. V. Astro Space Center of Lebedev Physical Institute 117810 Moscow, Russia

Komossa S. Astronomisches Institut Ruhr-Universität D-4630 Bochum, Germany

Kontorovich V. Institute of Radioastronomy Kharkiv 310002, Ukraine

Kopko M. Department of Physic \& Astronomy University of Pittsburg Pittsburgh, PA 15260, U.S.A.

Kovalev Y. A. Astro Space Center of Lebedev Physical Institute 117810 Moscow, Russia

Kovalev Y. Y. Astro Space Center \& Physical Dept. Moscow State University 117810 Moscow, Russia

Krichbaum T. Max-Planck-Institut für Radioastronomie D-5300 Bonn, Germany

Krolik J. H. John Hopkins University Dept. of Physics + Astronomy Baltimore, MD 21218, U.S.A.

Kukula M. J. Nuffield Radio Astronomy Laboratory GB-Macclesfield, Cheshire SK11 9DL, U.K.

Kurfess J. D. Naval Research Laboratory Code 4150 Washington, DC 20375, U.S.A.

Kusunose M. Copernicus Astronomical Center PL-00716 Warsaw, Poland

Lũdke E. University of Manchester Nuffield Radio Astr. Lab. GB-Macclesfield, Cheshire SK11 9DL, U.K. 
Lainela M. J. Tuorla Observatory SF-21500 Piikkiö, Finland

Lamer G. Astronomisches Institut Universität Tübingen D-7400 Tübingen, Germany

Lapidus J. I. Institute of Astronomy University of Cambridge GB-Cambridge, CB3 OHA, U.K.

Larionov G. M. Moscos State University Physical Department 119899 Moscow, Russia

Lawson A. University of Leicester GB-Leicester LEI JRH, U.K.

Leach C. M. Dept. of Physics Southampton University GB-Southampton, Hampsh. S09 5NH, U.K.

Lehnert M. D. IGPP Lawrence Livermore National Labs. Livermore, CA 94588, U.S.A.

Leighly K. Laboratory f. High Energy Astrophysics Greenbelt, MD 20771, U.S.A.

Leiter D. NASA-Goddard Space Flight Center Greenbelt, MD 20771, U.S.A.

Lichti G. Max-Planck-Institut für extraterrestrische Physik D-8046 Garching, Germany

Lindblad P. O. Stockholm Observatory S-13336 Saltsjöbaden, Sweden

Lipunov V. Sternberg Astronomical Institute 119899 Moscow, Russia

Litchfield S. J. Univ. of Central Lancashire Dept. of Physics \& Astronomy GB-Preston, PR1 2HE, U.K.

Macchetto F. Space Telescope Science Institute Baltimore, MD 21218, U.S.A.

Macchetto F. D. Space Telescope Science Institute Baltimore, MD 21218, U.S.A.

Machalski J. Jagiellonian University Astronomical Observatory PL-30244 Krakow, Poland

Maciołek-Niedźwiecki A. Nicolaus Copernicus Astronomical Center PL-00716 Warsaw, Poland

Madejski G. M. NASA-Goddard Space Flight Center Greenbelt, MD 20771, U.S.A.

Malkan M. Dept. of Astronomy UCLA Los Angeles, CA 90024-1562, U.S.A.

Mannheim K. Universitäts-Sternwarte D-3400 Göttingen, Germany

Maoz D. Institute for Advanced Study Princeton, NJ 08540, U.S.A.

Maraschi L. Istituto di Fisica I-20133 Milano, Italy

Marcha M. M. J. Univ. of Manchester NRAL Jodrell Bank GB-Macclesfield, Cheshire SK11 9DL, U.K.

Marcowith A. Laboratoire d'Astrophysique Observatoire de Grenoble F-38041 Grenoble Cedex, France

Marscher A. P. Dept. of Astronomy Boston University Boston, MA 02215-1401, U.S.A.

Marshall H. L. Center for Space Research Massachusetts Inst. of Technology Cambridge, MA 02139, U.S.A.

Massaro E. Istituto Astronomico Università di Roma "La Sapienza" I-00161 Roma, Italy

Mastichiadis A. Max-Planck-Institut für Kernphysik D-6900 Heidelberg, Germany

Mathur S. Harvard-Smithsonian Center for Astrophysics Cambridge, MA 02138, U.S.A.

Matt G. Institute of Astronomy University of Cambridge GB-Cambridge, CB3 OHA, U.K.

Mc Dowell J. C. Center for Astrophysics Cambridge, MA 02138, U.S.A.

Mc Hardy I. Astronomy \& Space Physics Group Southampton Univ. GB-Southampton, SO9 5NH, U.K.

Menon T. Max-Planck-Institut für Radioastronomie D-5300 Bonn, Germany

Mewe R. SRON Space Research Organization Netherlands NL-3584 CA Utrecht, The Netherlands

Meyer D. Dept. of Physics University of Michigan Ann Arbor, MI 48109-1090, U.S.A.

Meynet G. Observatoire de Genève 1290 Sauverny, Switzerland

Molthagen K. Hamburger Sternwarte Universität Hamburg D-21029 Hamburg, Germany

Moore E. Dept. of Astronomy Boston University Boston, MA 02215, U.S.A.

Moore N. P. Dept. of Physics \& Astronomy Univ. of Whales Coll. of Cardiff GB-Cardiff, CF2 3YB, U.K.

Mulchaey J. S. Space Telescope Science Institute Baltimore, MD 21218, U.S.A.

Mundell C. G. NRAL GB-Macclesfield, Cheshire SK11 9DL, U.K.

Naundorf C. E. Max-Planck-Institut für Radioastronomie D-5300 Bonn 1, Germany

Nazarova L. Royal Greenwich Observatory GB-Cambridge, CB3 OEZ, U.K.

Netzer H. School of Physics and Astronomy Tel-Aviv University Tel-Aviv 69978, Israel

Neumann M. Max-Planck-Institut für Astronomie D-6900 Heidelberg 1, Germany

Niemeyer M. Max-Planck-Institut für Radioastronomie D-5300 Bonn, Germany

Nolze W. Astronomisches Institut Westfälische Wilhelms-Univ. D-4400 Münster, Germany 
Nowak M. A. Canadian Institute of Theoretical Astrophysics Toronto, Ontario M5S 1A7, Canada

O'Brien P. T. Department of Physics \& Astronomy Univ. College London GB-London, WC1E 6BT, U.K.

Okayasu R. Nobeyama Radio Observatory Nagano, 384-13, Japan

Oknyanskij V. L. Sternberg Astronomical Institute 119899 Moscow, Russia

Orr A. Geneva Observatory 1290 Sauverny, Switzerland

Ozernoy L. M. Lab. de Radioastron. Millim. Ecole Normale Supérieure F-75231 Paris Cedex 05, France

Pacini F. Osservatorio Astrofisico di Arcetri I-50125 Firenze, Italy

Paltani S. Institut d'Astronomie de l'Université de Lausanne 1290 Chavannes-des-Bois,

Palumbo G. G. Università di Bologna Dipartimendo di Astronomia I-40126 Bologna, Italy

Papadakis I. Astronomy \& Space Physics Group Southampton Univ. GB-Southampton, S09 5NH, U.K.

Park M. Kyungpook Natl. University Dept. of Astronomy \& Meteorology Taegu 702-701, South Korea

Pastoriza M. G. Instituto de Fisica UFRGS Avenida Bento Goncalves 950091500 Porto Alegre, RS, Brasil

Pecontal E. Observatoire de Lyon F-69561 St Genis Laval Cedex, France

Pedlar A. University of Manchester NRAL, Jodrell Bank GB-Macclesfield, Cheshire SK11 9DL, U.K.

Pelletier G. Laboratoire d'Astrophysique Observatoire de Grenoble F-38041 Grenoble Cedex, France

Perez-Olea D. E. Dpto. de Física Teórica, C-XI. Universidad Autónoma de Madrid. E-28049 Madrid, Spain

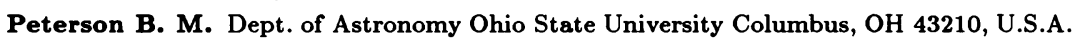

Petrini D. Observatoire de la Côte d'Azur F-06304 Nice Cedex, France

Pian E. Scuola Internazionale Sup. di Studi Avanzati I-34014 Trieste, Italy

Pietrini P. Dipartimento di Astronomia Universita di Firenze I-50125 Firenze, Italy

Piro L. Istituto Astrofisica Spaziale, CMNR Via E. Fermi 21 I-00044 Frascati, Italy

Pişmiş P. Instituto de Astronomia Universidad Nac. Auton. de Mexico Mexico, D.F. 04510, Mexico

Pogge R. W. Dept. of Astronomy Ohio State University Columbus, OH 43210-1106, U.S.A.

Poll A. Institut für Astrophysik und extraterrestrische Forschung D-53121 Bonn, Germany

Popescu N. A. Astronomical Institute of the Romanian Academy R-75212 Bucharest 28, Romania

Popovic L. Astronomical Observatory 11050 Belgrade, Yougoslavia

Poutanen J. Observatory and Astrophysics Laboratory, Univ. Helsinki SF-00014 Helsinki, Finland

Pronik I. 334413 Crimea, Ukraine

Röser H. Max-Planck-Institut für Astronomie D-6900 Heidelberg 1, Germany

Rafanelli P. Department of Astronomy University of Padova I-35122 Padova, Italy

Raikov A. St. Petersburg Indep. University SU-196135 St. Petersburg, Russia

Rantakyrö F. T. Onsala Space Observatory Göteborg University S-43992 Onsala, Sweden

Rees M. J. Institute of Astronomy GB-Cambridge, CB3 OHA, U.K.

Reichert G. A. Univ. Space Research Assoc. \& NASA GSFC Greenbelt, MD 20771, U.S.A.

Reinsch K. Universitäts-Sternwarte D-37083 Göttingen, Germany

Rigopoulou D. Queen Mary and Westfield College Dept. of Physics London E1 4NS, U.K.

Robson I. Joint Astronomy Centre Hilo, HI 96720, U.S.A.

Rodriguez P. M. IUE Observatory E-28080 Madrid, Spain

Rokaki E. Queen Mary and Westfield College Dept. of Physics GB-London, E1 4NS, U.K.

Romanova M. M. Space Research Inst. of the Russian Academy of Sciences 117810 Moscow, Russia

Saikia D. J. TATA Institute of Fundamental Research Pune 411007, India

Salamanca I. Observatoire de Meudon DAEC F-92195 Meudon Principal Cedex, France

Salas-Casales L. Instituto de Astronomia UNAM San Diego, CA 92143-9027, U.S.A.

Salvati M. Arcetri Astrophysical Observatory I-50125 Firenze, Italy

Sanders D. B. Institute of Astronomy Honolulu, HI 96822, U.S.A.

Sandqvist A. Stockholm Observatory S-13336 Saltsjöbaden, Sweden

Santos-Lleo M. Observatoire de Paris-Meudon DAEC F-92195 Meudon Principal Cedex, France 
Sapre A. K. Reader, School of Studies in Physics Pt. R.S. Shukla University Rajpur-492010, India Schalinski C. IRAM F-38406 Saint-Martin-d'Hères Cedex, France

Schlickeiser R. Max-Planck-Institut für Radioastronomie D-5300 Bonn, Germany

Schramm K. Institut d'Astrophysique Université de Liège B-4000 Liège, Belgique

Schulz H. Ruhr-Universität Astronomisches Institut D-4630 Bochum 1, Germany

Selvelli P. CNR Osservatorio Astronomico di Trieste I-34131 Trieste, Italy

Setti G. Istituto di Radioastronomia I-40126 Bologna, Italy

Shevchenko I. I. Inst. of Theoret. Astronomy Russian Academy of Sciences SU-191187 St. Petersburg, Russ

Shields J. C. Dept. of Astronomy Ohio State University Columbus, OH 43210, U.S.A.

Sillanpaa A. Tuorla Observatory SF-21500 Piikkiö, Finland

Simpson C. Dept. of Astrophysics Oxford University GB-Oxford, OX1 3RH, U.K.

Sol H. Observatoire de Meudon F-92195 Meudon Principal Cedex, France

Solomos N. Hellenic Naval Academy Dept. of Physics GR-18503 Piraeus, Greece

Staubert R. Astronomisches Institut Universität Tübingen D-7400 Tübingen, Germany

Steffen W. Max-Planck-Institut für Radioastronomie D-5300 Bonn 1, Germany

Stenholm L. Uppsala Observatory S-75120 Uppsala, Sweden

Stevens J. A. University of Central Lancashire Dept. of Physics and Astronomy Preston PR1 2HE, U.K.

Stift M. J. Institut für Astronomie voir adresses vacances aout dans dossier A-1180 Wien, Austria

Stirpe G. M. Osservatorio Astronomico di Bologna I-40126 Bologna, Italy

Storchi-Bergmann T. Instituto de Fisica UFRGS Av. Bento Goncalves 950091500 Porto Alegre, RS, Bras Sun W. Institute of Astronomy Natl. Central University Chung-Li, 320 ROC, Taiwan

Suran D. M. Astronomical Institute of the Romanian Academy R-75212 Bucharest 28, Romania

Svensson R. Stockholm Observatory S-13336 Saltsjöbaden, Sweden

Swings J. Institut d'Astrophysique Université de Liège B-4000 Liège, Belgique

Tacconi-Garman L. E. Max-Planck-Institut für extraterrestrische Physik D-8046 Garching, Germany

Takalo L. O. Tuorla Observatory SF-21500 Piikkiö, Finland

Tanaka Y. Institute of Space and Astronautical Science Kanagawa-Ken 229, Japan

Tanzi E. Istituto di Fisica Cosmica CNR I-20133 Milano, Italy

Tashiro M. Dept. of Physics University of Tokyo Tokyo, 113, Japan

Teräsranta H. Metsähovi Radio Research Station SF-02540 Kylmälä, Finland

Thomas H. Max-Planck-Institut für Astrophysik D-85740 Garching, Germany

Thompson D. J. NASA Goddard Space Flight Center Greenbelt, MD 20771, U.S.A.

Thompson R. Steward Observatory University of Arizona Tucson, AZ 85721, U.S.A.

Tornikoski M. Metsähovi Radio Research Station SF-02540 Kylmälä, Finland

Torricelli G. Observatoire de Genève 1290 Sauverny,

Tovmassian H. Instituto Nacional de Astrofisica Optica y Electronica 72000 Puebla, Mexico

Trevese D. Istituto Astronomico Università di Roma "La Sapienza" I-00161 Roma, Italy

Trussoni E. Osservatorio Astronomico di Torino I-10025 Pino Torinese, Italy

Tsvetanov Z. Center for Astrophysical Sciences John Hopkins University Baltimore, MD 21218, U.S.A.

Tytler D. University of California, San Diego La Jolla, CA 92093-0111, U.S.A.

Ulrich M. European Southern Observatory D-8046 Garching, Germany

Valtaoja E. Metsähovi Radio Research Station SF-02540 Kylmala, Finland

Valtaoja L. NORDITA Nordisk Institut for Teoretisk Fysik DK-2100 Copenhagen 0, Denmark

Van Groningen E. Astronomiska Observatoriet S-75120 Uppsala, Sweden

Varshalovich D. A. A.F. Ioffe Inst. Phys. and Technology, SU-194021 St. Petersburg, Russia

Vicente L. Observatoire de Meudon DARC F-92195 Meudon Cedex, France

Vio R. Villa Franca del Castillo Satellite Tracking Station E-28080 Madrid, Spain 
Vogel S. Hamburger Sternwarte Universität Hamburg D-2050 Hamburg 80, Germany Von Linde J. Hamburger Sternwarte Universität Hamburg D-21029 Hamburg, Germany Wagner S. J. Landessternwarte Königstuhl D-6900 Heidelberg, Germany Wallinder F. NORDITA Nordisk Institut for Teoretisk Fysik DK-2100 Copenhagen, Denmark Walter R. Max-Planck-Institut für Extraterrestrische Physik D-8046 Garching, Germany Wamsteker W. VILSPA ESA IUE Observatory E-28080 Madrid, Spain

Wandel A. Observatoire de Paris F-92195 Meudon, France

Wanders I. Astronomiska Observatoriet S-75120 Uppsala, Sweden

Wang Y. Acton, MA 01720, U.S.A.

Ward M. J. Oxford University Astrophysics GB-Oxford, OX1 3RH, U.K.

Warwick R. S. Dept. Physics and Astronomy University of Leicester GB-Leicester, LEI 7RH, U.K.

Webb J. R. Dept. of Physics Florida Intl. University Miami, FL 33199, U.S.A.

Wei C. Center for Astrophysics University of Science and Technology 230026 Hefei, Anhui, China

Wiita P. J. Dept. of Physics and Astronomy Georgia State University Atlanta, GA 30303-3083, U.S.A.

Winge C. Dept. of Astronomy The Ohio State University Columbus, OH 43210, U.S.A.

Wisotzki L. Hamburger Sternwarte Universität Hamburg D-2050 Hamburg 80, Germany

Woltjer L. Observatoire de Haute-Provence F-04870 St Michel-l'Observatoire, France

Worrall D. M. Harvard-Smithsonian Center for Astrophysics Cambridge, MA 02138, U.S.A.

Yankulova I. M. Dept. of Astronomy Sofia University BG-1126 Sofia, Bulgaria

Yi I. Harvard-Smithsonian Center for Astrophysics, MS 51 Cambridge, MA 02138, U.S.A.

Zdziarski A. Copernicus Astronomical Center PL-00716 Warsaw, Poland

Zensus A. N.R.A.O. Socorro, NM 87801, U.S.A.

Zycki P. Nicolaus Copernicus Astronomical Center PL-00716 Warszawa, Poland 